\title{
OBJECT FIXATION BY THE BLOWFLY DURING TETHERED FLIGHT IN A SIMULATED THREE-DIMENSIONAL ENVIRONMENT
}

\author{
BERND KIMMERLE*, JUDITH EICKERMANN AND MARTIN EGELHAAF \\ Lehrstuhl für Neurobiologie, Fakultät für Biologie, Universität Bielefeld, Postfach 1001 31, D-33501 Bielefeld, \\ Germany \\ *Present address: Institut für Neurobiologie, Freie Universität Berlin, Königin-Luise-Straße 28-30, D-14195 Berlin, Germany \\ (e-mail: kimmerle@zedat.fu-berlin.de)
}

Accepted 21 March; published on WWW 10 May 2000

\begin{abstract}
Summary
The ability of flies to detect and fixate objects moving relative to their background was investigated in a flight simulator during translational tethered flight. The fly experienced optic flow that depended on its own actions and reactions in a similar way as in free-flight (closed-loop) conditions. Fixation of an object required turning responses towards it. The simulated distances between the

part of the fly's visual field. Fixation responses were only elicited when the object was simulated to be closer than the background. The fly's fixation performance was better with close than with more distant objects. Since, under many stimulus conditions, fixation responses were either elicited or entirely failed to be elicited, it is concluded that object fixation behaviour is gated in the visuo-motor pathway.
\end{abstract} fly, object and background were varied systematically by changing the velocities with which the object and the background pattern moved from the frontal to the back

Key words: flight, object fixation, vision, optic flow, closed loop, blowfly, Lucilia sp.

\section{Introduction}

During locomotion, the visual system is subjected to continuous changes of the retinal images termed 'optic flow'. Turns to one side are accompanied by motion of the whole retinal image in the opposite direction. Optic flow elicited during rotation around a body axis is independent of the distance of the objects in the visual surround. In contrast, during translation, the retinal image of an object moves faster than the retinal image of its background. Thus, the retinal images of object and background move relative to each other.

Visual systems have developed strategies to use the information provided by optic flow for different orientation tasks. Many animals compensate global rotational image motion around a body axis by eye, head and/or body movements in the opposite direction. These optokinetic or optomotor responses serve to stabilize the whole retinal image or at least part of it (Miles and Wallman, 1993) and have been interpreted in insects as a mechanism to compensate for unintended deviations from a straight path of locomotion. Compensatory optomotor responses have been studied in great detail in the fly (e.g. Fermi and Reichardt, 1963; Götz, 1964, 1975; Wolf and Heisenberg, 1990). Relative motion in the retinal image, in contrast, signals the presence of an object in the visual field. Relative motion cues can therefore be used to discriminate an object from its background. Detection and fixation of objects solely defined by relative motion have been investigated, for example, in humans (e.g. van Doorn and Koenderink, 1982, 1983; Regan and Beverly, 1984), in monkeys (e.g. Miles and Kawano, 1987), in bees (e.g. Srinivasan et al., 1990; Kern et al., 1997) and in flies (Virsik and Reichardt, 1976; Reichardt et al., 1983; Egelhaaf, 1985a; Kimmerle et al., 1996, 1997).

In flies, flight control and visual orientation can be investigated on different levels ranging from free-flight behaviour to the neuronal and subcellular levels (for reviews, see Egelhaaf and Borst, 1993; Egelhaaf and Warzecha, 1999). Both optomotor turning behaviour and fixation responses can be investigated under controlled stimulus conditions using tethered flies in a flight simulator. In previous studies using the flight simulator, flies were shown to fixate an object in the frontal part of their visual field even if the object could be discriminated from the background only by means of relative motion (Virsik and Reichardt, 1976; Reichardt et al., 1983; Egelhaaf, 1985a). These experiments were performed in a cylindrical arena in which both the object and the background could be rotated only around the fly's vertical body axis. Hence, flight situations in an environment were simulated in which no translational optic flow was present, corresponding to a situation in which object and background were at an infinite distance from the fly. In a realistic stationary three-dimensional environment, rotation of the animal around one of its body axes alone does not provide any relative motion cues. Therefore, the turning responses of tethered flies to objects defined by relative motion were subsequently investigated during simulated translational flight (Kimmerle et al., 1997). However, in these 
experiments, the turning responses of the fly had no influence on the visual input, which was exclusively determined by the experimenter ('open-loop' conditions). Of course, this is different from the natural flight situation in which the turning responses of the fly immediately affect the retinal input ('closed-loop' conditions). In the present study, object detection during simulated translational flight was analyzed under closedloop conditions in which the turning responses of the fly were coupled to the rotational component of the motion stimuli. The fly's turning behaviour therefore influenced the optic flow experienced by the animal in a manner corresponding to situations in unrestrained flight. Object fixation was investigated while systematically varying the simulated distances between the fly and an object and the background.

\section{Materials and methods \\ Animal preparation}

Female blowflies of the genus Lucilia, bred from our laboratory stocks, were briefly anaesthetized either with $\mathrm{CO}_{2}$ or by cooling them on ice. Using beeswax, the head was fixed to the thorax, and a small triangular piece of cardboard that suspended the fly from the torque compensator was glued onto the pronotum (Fig. 1, inset).

\section{Visual stimuli and acquisition of behavioural responses}

Visual stimuli were presented on a circular arena of lightemitting diodes (LEDs) designed and built at the University of Bielefeld, Germany (Fig. 1). The arena consisted of 10 identical circuit boards. Each board contained 48 columns and 30 rows of single LEDs $(5 \mathrm{~mm} \times 2.5 \mathrm{~mm}$, green $)$. The LEDs were soldered to the boards in such a way that the surface of the array was slightly curved along the horizontal axis. The vertical columns of the array could be switched on or off independently. The time until an LED reached a constant luminance value after switching on or off was $20-50 \mu$ s. Stripe patterns were generated using two eight-bit registers connected to two parallel ports of a computer, one specifying the state of eight neighbouring LED columns ('on' or 'off'), the other specifying the respective board and the location of these columns on the board. Generating one frame, i.e. addressing 60 groups of eight columns serially, took approximately $370 \mu \mathrm{s}$. The luminance of the bright LEDs when switched on was 500-900 $\mathrm{cd} \mathrm{m}^{-2}$; when the LEDs were switched off, it was approximately $20 \mathrm{~cd} \mathrm{~m}^{-2}$. The arena had a diameter of $37 \mathrm{~cm}$ and a height of $15 \mathrm{~cm}$. When viewed from the centre, it therefore had a vertical extent of $\pm 22^{\circ}$. The horizontal angular extent of each LED column was $0.75^{\circ}$.

Periodic square-wave gratings with a spatial wavelength of $7.5^{\circ}$ were displayed on the LED arena. Optic flow simulating flight situations in which the fly translates along its longitudinal axis and turns around its vertical body axis was achieved in the following way (Fig. 1). The fly was suspended from a torque meter measuring the turning responses of the tethered flying animal around its vertical body axis (Fermi and Reichardt, 1963; Götz, 1964). Data aquisition and the visual stimuli were
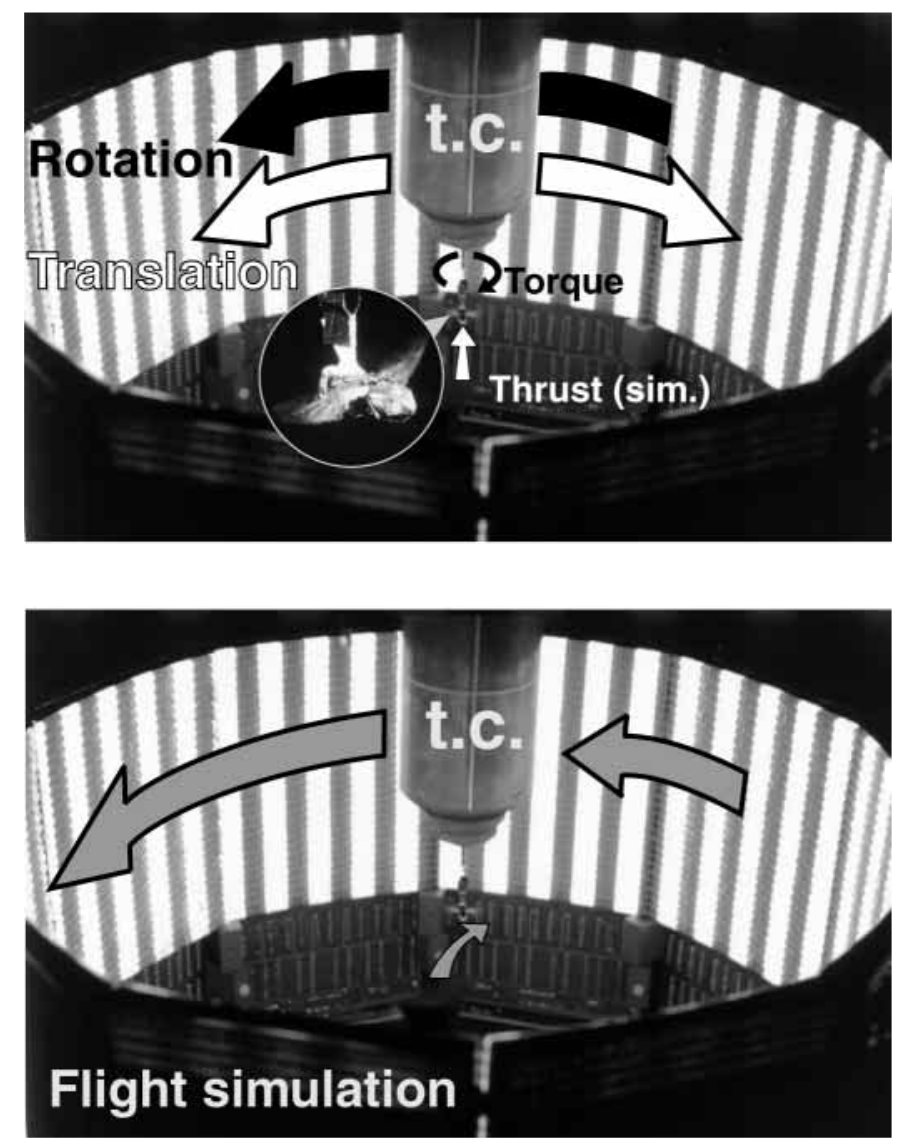

Fig. 1. Generation of optic flow in the flight simulator. Top: Rotational and translational components of optic flow. A fly was suspended from a torque compensator (t.c.) in the centre of a cylindrical arena of light-emitting diodes. The inset shows an enlarged lateral view of the tethered fly. The torque produced by the fly around its vertical body axis (small black arrow) was measured continuously and coupled to the rotational velocity of the grating displayed in the arena (large black arrow). Turning responses in the clockwise direction caused counterclockwise pattern motion and vice versa. The translational flight force along the fly's longitudinal body axis (thrust, small white arrow) was not measured; instead, the consequences of translation for the visual input of the fly were simulated by constant-velocity motion of the grating from front to back in the right and left halves of the arena (large white arrows). Bottom: Compound stimulus. The rotational and translational components of the optic flow were superimposed in each half of the arena (large grey arrows), thereby simulating flight situations in which the fly was moving along a curved path (small grey arrow).

computer-controlled via an I/O card (Data Translation DT2801A) and the parallel ports of a computer. The sampling rate and the rate at which the frames in the arena were generated were $200 \mathrm{~Hz}$. The torque signal was coupled to the rotational velocity of the grating such that a torque of $10^{-7} \mathrm{~N} \mathrm{~m}$ resulted in a pattern velocity of $15.5^{\circ} \mathrm{s}^{-1}$ in the opposite direction. Translation was simulated by front-to-back motion at a constant velocity in the right and left visual fields of the fly. The rotational and translational velocities were added in both halves of the visual field. 
An object could be superimposed on the background grating (see Fig. 2A). The pattern on the object exactly matched that on the background. The object covered the entire vertical extent of the arena and had a width of either $15^{\circ}$ ('small object') or $60^{\circ}$ ('large object'). The rotational velocities of the object and background were determined by the turning responses of the fly in the same way. Rotation alone, therefore, did not induce relative motion between object and background. By adding a constant external motion bias always directed from front to back to the object, translation of the fly alongside the object was simulated. By varying the velocity of the superimposed motion bias (translational velocity), different distances of the object were simulated. Different distances of the background were simulated by varying the velocity of a front-to-back motion bias added independently from object translation in both the right and left halves of the background pattern (background translation). The translational velocities used and the terminology according to which the respective simulated distances will be referred to are as follows: $0^{\circ} \mathrm{s}^{-1}$ ('infinite'), $15^{\circ} \mathrm{s}^{-1}$ ('distant'), $60^{\circ} \mathrm{s}^{-1}$ ('close') and $240^{\circ} \mathrm{s}^{-1}$ ('very close'). The translational motion bias of the object was always directed from front to back; it was positive by definition when the object was in the right part of the visual field and negative when it was in the left part of the visual field. Stimulus conditions were also presented in which the background moved at a higher velocity than the object, simulating a situation in which the background is closer than the object. Although, under these conditions, the background would better be referred to as foreground, for convenience we use the term 'background' irrespective of its simulated distance.

\section{Experimental design}

Because of possible deviations from exact suspension of the fly in the flight simulator and possible asymmetries in the flight motor, the reference torque corresponding to straight flight had to be determined at the beginning of an experiment. This procedure was based on the assumption that the fly's compensatory optomotor response is symmetrical, i.e. clockwise and counterclockwise pattern rotation at a given velocity lead to the same average torque in the respective direction. The mean torque during clockwise and counterclockwise pattern motion was therefore assumed to correspond to the zero (reference) level. This value was determined in an iterative manner. First, an arbitrary value was assumed as the reference torque. The torque of the tethered flying fly was coupled to the rotational velocity of the whole grating, and a constant rotational motion bias was superimposed. No translation was added, and no object was displayed. The direction of the motion bias was switched between clockwise and counterclockwise every $5 \mathrm{~s}$. The torque produced by the fly was averaged over periods of $10 \mathrm{~s}$. This value was then used as the new reference torque for the next $10 \mathrm{~s}$ period. After five repetitions, the final two reference values were averaged and taken as the reference torque for the experimental trials.

Object fixation trials lasted $20 \mathrm{~s}$. Only background motion was presented for the first $5 \mathrm{~s}$. The object was then introduced in front of the fly $\left(0^{\circ}\right.$ by definition). In subsequent trials, any combination of the four different translational velocities of object and background (see above) was used except for those yielding no relative motion (identical translational velocities of object and background). In further trials, object motion at the four different velocities was displayed while the background was stationary. Each of these conditions was tested with the small and the large object. In total, 32 different stimulus conditions were presented in pseudorandom order. Each condition was tested 39 times with a total of 36 flies. Each fly was tested with the complete set of stimulus conditions once or, at most, twice. All the data presented below were taken from this set of experiments.

\section{Results}

Flies were able to fixate objects if the objects moved relative to their background. Two examples of fixation responses are shown in Fig. 2. In the first example (Fig. 2Bi-Di), the background was simulated to be at infinite distance and the object close to the fly. In the second example (Fig. 2Bii-Dii), the background was simulated to be distant and the object very close to the fly. During the initial periods in which no object was present, the torque fluctuated around zero, indicating that the fly constantly changed its intended direction of flight, but that, on average, it flew straight ahead (Fig. 2Bi,ii). At the instant when an object was introduced in front of the animal, the fly was generating a small torque in the counterclockwise direction in both examples, so the object was shifted clockwise into the right visual field of the fly (Fig. 2Ci,ii). This can be compared with the free-flight situation during a left turn of the fly. Without a further turning responses, the object would have moved with a constant velocity out of the frontal region of the visual field because of its translational motion bias (dotted lines in Fig. 2Ci,ii). However, immediately after the object appeared, the fly tried to turn towards it. Repeated turning responses in the clockwise direction were observed in the first example (Fig. 2Bi) and a short, strong turning response was recorded in the second example (Fig. 2Bii). As a consequence of the turning responses in the first example, the object did not move into the rear part of the visual field but was fixated in the fronto-lateral part (Fig. 2Ci). In the second example, the object was not fixated but decelerated for a short period (Fig. 2Cii). Before the object appeared, the background velocity in the first example fluctuated around zero, because the average torque elicited by the fly was zero and because the background was simulated to be at infinite distance and therefore did not lead to translational image flow (Fig. 2Di). After the object had appeared, the fly's turning response towards the object caused a drift of the background in the opposite direction. In the second example, the background was simulated to be at a finite distance, and the velocities in the right and left visual fields therefore differed from each other because of the translational velocity component (Fig. 2Dii). Before the object appeared, the background was moving from front to back in both visual 

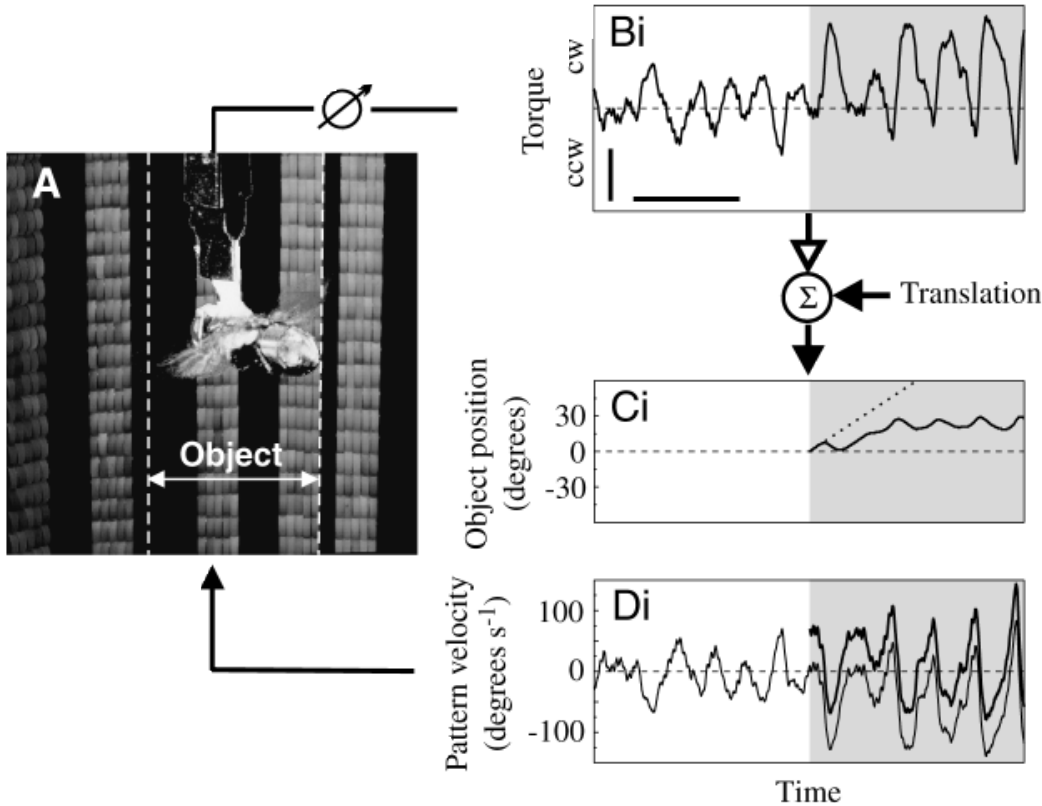

Behavioural response
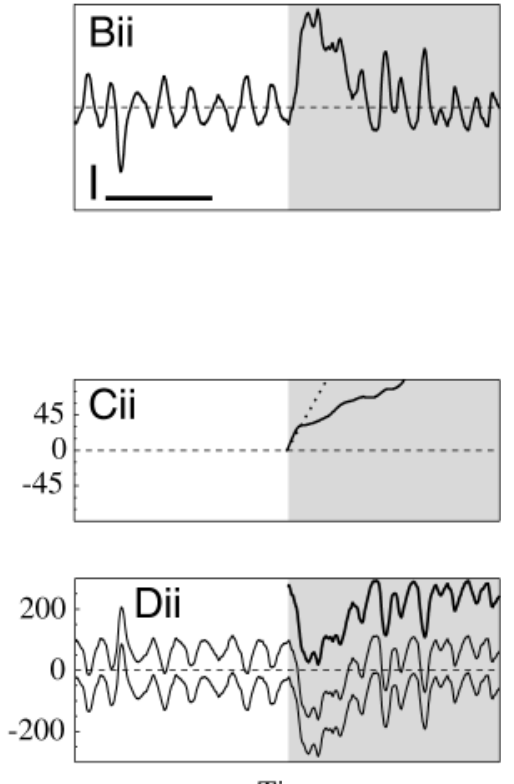

Time

Fig. 2. Object fixation under closed-loop conditions. Two examples of object fixation with the object and background at different simulated distances from the fly: a close object and infinitely distant background (Bi-Di); and a very close object and distant background (Bii-Dii). See Materials and methods for definitions of object distances. (A) Close-up of the tethered flying fly. The horizontal extent $\left(15^{\circ}\right)$ of a small object superimposed on the background grating is indicated by the white arrow and the dashed lines. (Bi,ii) Torque elicited by the fly prior to (white area) and after (shaded area) the appearance of the object. The traces show only a section of the recordings. Vertical scale bars, $5 \times 10^{-7} \mathrm{~N} \mathrm{~m}$; horizontal scale bars, $1 \mathrm{~s}$. The torque signal (cw, clockwise; ccw, counterclockwise) was coupled to the rotational components of object and background motion. Translation of the fly was simulated by adding front-to-back pattern motion. (Ci,ii) Time course of the position of the object. The same time intervals are shown as in Bi,ii. Dotted lines indicate the position the object would have taken under open-loop conditions. (Di,ii) Time course of the velocities of the object (thick lines) and background (thin lines). In the example shown in Bii-Dii, the background was simulated at a finite distance by adding a motion bias, so the background velocity in the right half of the arena (Dii, upper thin line) differed from that in the left half of the arena (Dii, lower thin line).

fields for most of the time, although at continuously changing velocities. During the strong object-directed turning response, the front-to-back motion of the object was almost compensated for a short period, whereas the background drifted in the opposite direction at high velocity in both parts of the visual field (Fig. 2Dii).

\section{Torque fluctuations before the object appeared}

The examples shown in Fig. 2 illustrate that, in the absence of an object, the flies flew on average straight ahead, although the torque produced by the fly fluctuated continuously around zero. The amplitude of the torque fluctuations before the appearance of the object depended either on whether the background was stationary, i.e. no feedback of the turning behaviour on the visual input was present (open-loop situation), or on whether the turning responses were fed back to the visual input (closed-loop situation) (Fig. 3). In the latter case, the strength of the added translational motion bias, and therefore the simulated distance of the background, influenced the amplitude of the torque fluctuations elicited by the fly. The most pronounced torque fluctuations prior to object appearance were generated when the background was simulated to be very close. In this case, the fluctuations were stronger than the open-loop torque fluctuations. In all other cases, the closed-loop situation led to smaller fluctuations than were obtained under open-loop conditions. The smallest fluctuations in the turning responses

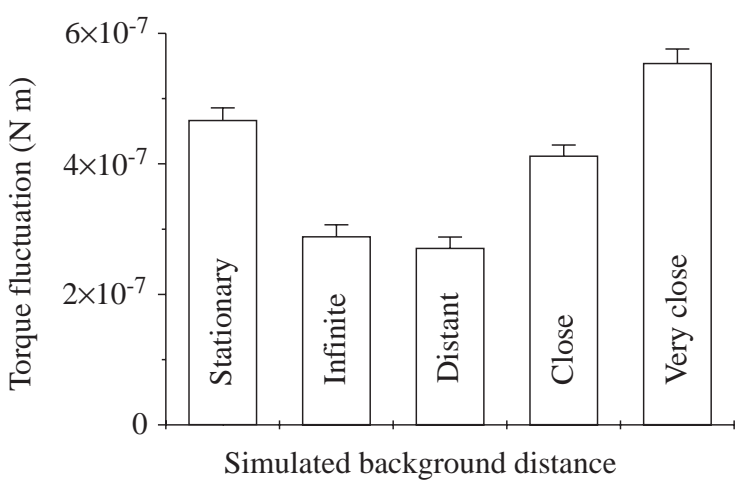

Fig. 3. Torque fluctuations in the absence of an object. The standard deviations of the torque produced by the fly during the last $3 \mathrm{~s}$ prior to object appearance were averaged over all trials sharing the same simulated background distance. $N=312$ (stationary background); $N=234$ (other stimulus conditions). Error bars denote S.E.M. See Materials and methods for definitions of stimulus conditions. 
around a straight flight course were observed when the background was simulated to be distant or at infinite distance.

The finding that visual feedback reduces the torque fluctuations compared with flight under open-loop conditions is in accordance with previous experiments. Heisenberg and Wolf (1988) showed that the fluctuations of optomotor turning responses in Drosophila melanogaster are greater under openloop than under closed-loop conditions. The present results extend this finding with respect to the influence of concurrent translational motion. They indicate that the smallest torque fluctuations, and therefore the most effective stabilization of the retinal image, are achieved when the background is either not translating or translating only slowly, i.e. during flight distant from the background.

\section{Influence of the simulated distances of object and background on fixation}

How well large and small objects could be fixated by the fly depended on the simulated distances of the object and background (Fig. 4). The histograms show the probability distributions of the positions of the object during the first $3 \mathrm{~s}$ after the object appeared in front of the fly. Position histograms are shown for all combinations of simulated object and background distances and for conditions in which the turning responses were not fed back to background motion. In general, fixation of the object can be inferred from peaks in the position histogram, indicating that the object stayed preferentially in a particular area of the visual field. Object position probabilities need to be compared with the reference probability that would have been obtained if the object had moved from front to back without being coupled to the turning responses of the fly (solid lines in Fig. 4B-D). The reference position probabilities for infinitely distant objects (no translation) were determined by assuming the presence of an object during $3 \mathrm{~s}$ before it actually appeared and by calculating post hoc the position probability for such a hypothetical object (not shown).

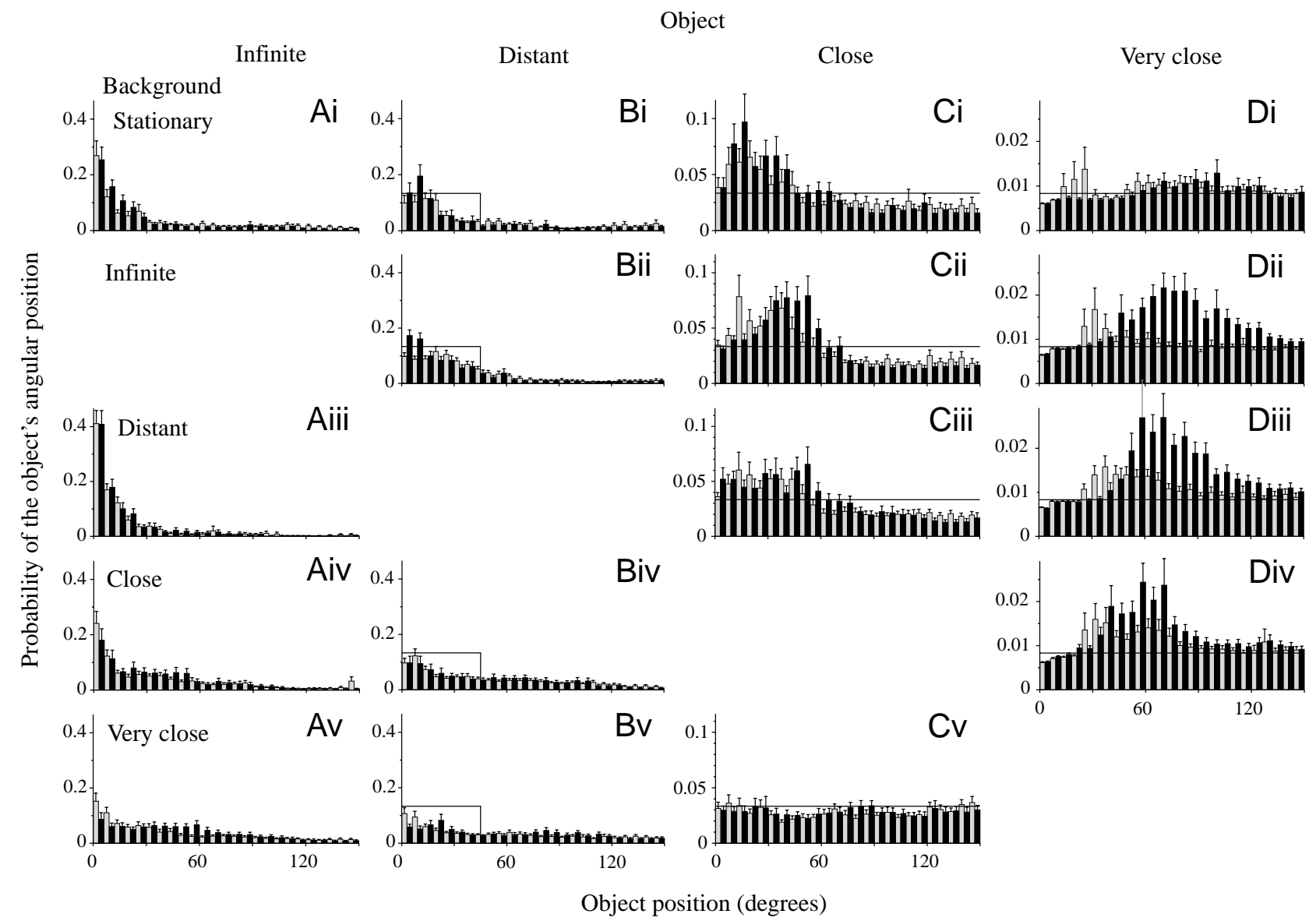

Fig. 4. Dependence of object fixation on the simulated distances of the object and background from the fly. Probability of the object's angular position for a small (grey columns) object and a large (black columns) object during the first $3 \mathrm{~s}$ after its appearance. Corresponding object positions in the right and left visual fields were pooled. The spatial resolution was $6^{\circ}$ (to visualize both distributions, the columns are drawn with a width of $3^{\circ}$ ). Histograms obtained with the same background motion condition are aligned in rows, those obtained with the same translational velocity of object motion are aligned in columns. The integrals of the probabilities from 0 to $180^{\circ}$ sum to 1 for each distribution. Close and very close objects remained at the back $\left(180^{\circ}\right)$ with the highest probability (not shown). Straight lines in B-D represent the position probabilities of the object under open-loop conditions. Values are means + S.E.M., $N=39$. See Materials and methods for definitions of stimulus conditions. See text for further explanation. 
Table 1. Fixation probabilities that an object remained within the area indicated

\begin{tabular}{|c|c|c|c|c|c|c|}
\hline \multirow[b]{2}{*}{ Background } & \multicolumn{2}{|c|}{ Object distant (less than $\pm 12^{\circ}$ ) } & \multicolumn{2}{|c|}{ Object close (less than $\pm 60^{\circ}$ ) } & \multicolumn{2}{|c|}{ Object very close (less than $\pm 120^{\circ}$ ) } \\
\hline & Small object & Large object & Small object & Large object & Small object & Large object \\
\hline Stationary & $0.203 \pm 0.037$ & $0.331 \pm 0.056$ & $0.451 \pm 0.053$ & $0.561 \pm 0.054$ & $0.188 \pm 0.020$ & $0.178 \pm 0.014$ \\
\hline Infinite & $0.186 \pm 0.022$ & $0.334 \pm 0.038$ & $0.520 \pm 0.054$ & $0.568 \pm 0.050$ & $0.187 \pm 0.018$ & $0.282 \pm 0.022$ \\
\hline Distant & & & $0.468 \pm 0.049$ & $0.512 \pm 0.051$ & $0.218 \pm 0.017$ & $0.303 \pm 0.028$ \\
\hline Close & $0.220 \pm 0.035$ & $0.193 \pm 0.040$ & & & $0.214 \pm 0.019$ & $0.268 \pm 0.021$ \\
\hline Very close & $0.202 \pm 0.039$ & $0.111 \pm 0.018$ & $0.274 \pm 0.029$ & $0.279 \pm 0.032$ & & \\
\hline Open-loop & \multicolumn{2}{|c|}{0.267} & \multicolumn{2}{|c|}{0.333} & \multicolumn{2}{|c|}{0.167} \\
\hline
\end{tabular}

Values are the mean \pm S.E.M. $(N=29)$ of the probability that the object remained within the indicated area during the first $3 \mathrm{~s}$ after it appeared. The bottom row gives the probability with which an object moving under open-loop conditions would have remained in the respective area. See Materials and methods for definition of stimulus conditions.

Fixation of an object simulated to be infinitely distant (Fig. 4A) seemed to be best in front of a distant background (Fig. 4Aiii) and worst in front of a very close background (Fig. 4Av). However, the turning behaviour of the fly in the absence of an object would have led to very similar position probabilities to those obtained when an object was present (not shown). The only case in which the object was fixated in the frontal part of the visual field (less than $\pm 12^{\circ}$ ) with a higher probability than the reference probability derived from flight behaviour before the appearance of the object was the situation in which the background was stationary (Fig. $4 \mathrm{Ai} ; P<0.05$ for the small object, $P<0.001$ for the large object; Wilcoxon matched-pairs test). In other words, 'fixation' in the situations with a moving background and an infinitely distant object was only the consequence of the flight course the fly would have taken even if there had been no object. Real fixation of an infinitely distant object was therefore apparent only when the background was stationary.

Distant objects did not seem to be fixated (Fig. 4B). The probability of the object remaining in the frontal part of the visual field (less than $\pm 12^{\circ}$ ) was in no case significantly larger than it would have been under open-loop conditions (Table 1; Wilcoxon matched-pairs test). Despite these conclusions based on average data, both the small and the large object were fixated in some of the trials during which the background was stationary or at infinite distance (Fig. 4Bi,ii), as will be shown below.

When the object was simulated to be increasingly close, its preferred position was shifted towards increasingly lateral parts in the visual field (Fig. 4A-D). Close objects were fixated at positions more frontal than $\pm 60^{\circ}$ (Fig. 4C). The probability of the object remaining within this region was larger than under open-loop conditions if the object was large and the background was not simulated to be very close (Fig. 4Ci-iii; Table 1; $P<0.01$ for each condition, Wilcoxon matched-pairs test). Fixation of the small object at positions more frontal than $\pm 60^{\circ}$ was only significant if the background was infinitely distant (Fig. 4Cii; Table 1; $P<0.05$, Wilcoxon matched-pairs test). However, the probability of a position more frontal than $\pm 60^{\circ}$ was not significantly different for a small and for a large object in any of the conditions, indicating that small objects were also fixated in some of the trials. No fixation responses were elicited when the background was simulated to be very close (Fig. 4Cv).

Very close objects were fixated at positions more frontal than $\pm 120^{\circ}$ with a higher probability than under open-loop conditions when they were large and when the background was non-stationary (Fig. 4D; Table 1; $P<0.001$ for all conditions, Wilcoxon matched-pairs test). Fixation of the small object was only significant if the background was distant and positions more frontal than $90^{\circ}$ were considered $(P<0.05$, Wilcoxon matched-pairs test). The large object was fixated at a position more frontal than $120^{\circ}$ with a higher probability than the small object in all conditions except with a stationary background $(P<0.05$, conditions shown in Fig. 4Dii-iv; Wilcoxon matched-pairs test).

In conclusion, object fixation was best when the object was simulated to be close or very close to the fly. Generally, when the background was simulated to be closer than the object, no fixation responses were elicited. With increasing simulated proximity between the object and the fly, the object was fixated at an increasingly lateral position. The large object was fixated more readily by the fly than the small object, the latter tending to be fixated more frontally than the large object. Background motion enhanced fixation of large objects when the object was simulated to be very close.

\section{Spatio-temporal aspects of object fixation}

The probability distributions of object positions shown in Fig. 4 do not allow an assessment to be made of how the fixation responses change over time. Therefore, the probabilities with which the object stayed at a given position in the visual field during a particular time bin are shown for some of the stimulus conditions in Figs 5 and 6. A scrutiny of these spatio-temporal probability distributions revealed additional features of the fly's fixation responses that are not obvious from in the purely spatial probability distributions.

The spatial probability distributions (Fig. 4) did not indicate that small distant objects could be fixated by the fly. However, inspection of the spatio-temporal distributions revealed that both large and small distant objects could be fixated if presented in front of a stationary background (Fig. 5A,B). For small objects, a distinction into two types of reactions became 
Fig. 5. Spatio-temporal probability distributions of object position for a distant object. The translational velocity of the object was $15^{\circ} \mathrm{s}^{-1}$. (A,B) Stationary background. (C,D) Background simulated at infinite distance. Object width was $15^{\circ}(\mathrm{A}, \mathrm{C})$ and $60^{\circ}(\mathrm{B}, \mathrm{D})$. The time axes of the spatio-temporal probability maps start with the appearance of the object and end $10 \mathrm{~s}$ later. Horizontal scale bar, $2 \mathrm{~s}$. The position axes start at $0^{\circ}$ (bottom of the diagrams) and end at $180^{\circ}$. Vertical scale bar, $30^{\circ}$. Data obtained for objects moving in the left half of the visual field were mirror-inverted and pooled with data obtained for objects moving in the right half of the visual field. Dashed lines indicate the course the object would have taken under open-loop conditions. The resolution is $200 \mathrm{~ms}$ in time and $6^{\circ}$ in space. Grey shading indicates the probability of the object being at a particular position within a given time interval and is incremented in six linear steps. Black corresponds to one-quarter of the maximal probability that a spatio-temporal bin could obtain if the object were to move under open-loop onditions. $N=39$. See Materials and methods for definitions of stimulus conditions.

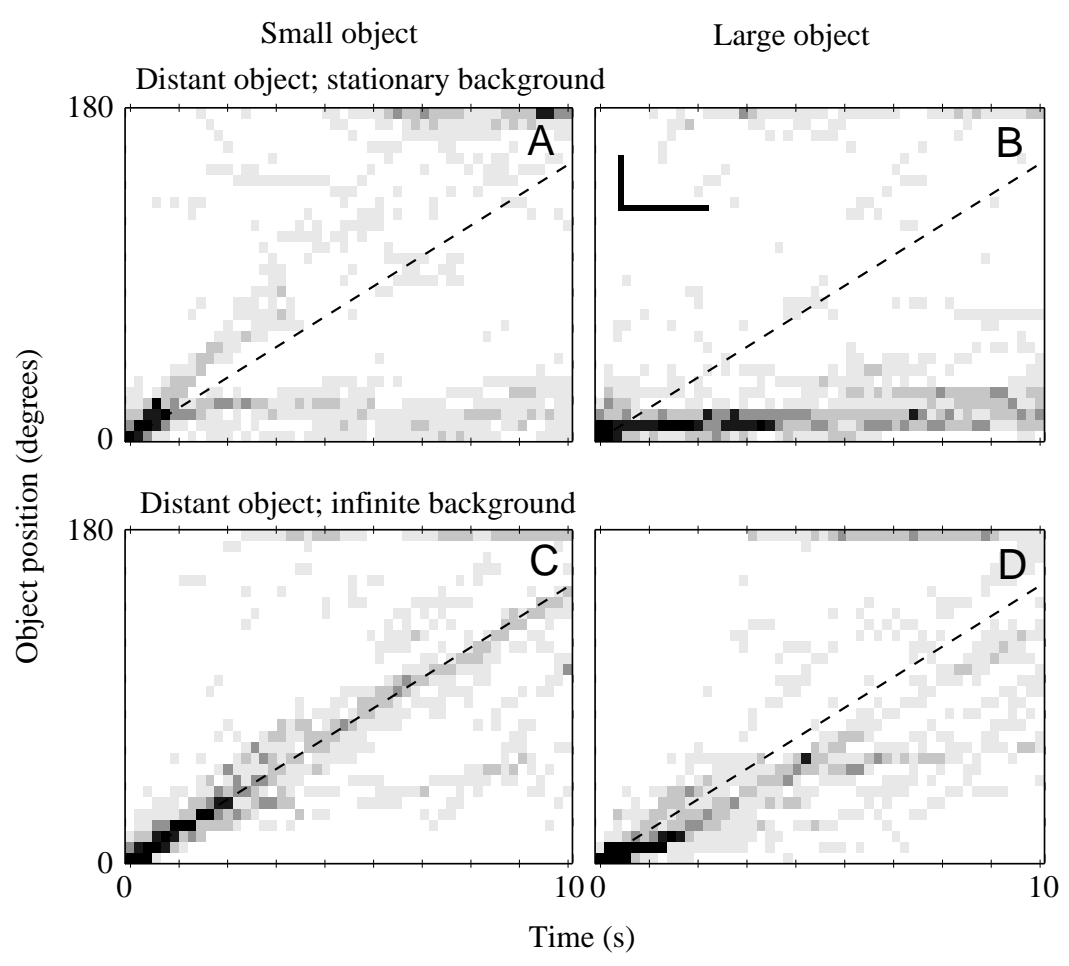

When the background was simulated to be at infinite distance from the fly (Fig. 5C,D), the small object most frequently moved from front to back at its open-loop velocity and, therefore, did not affect the flight course of the fly. Object fixation was less probable but occurred occasionally, indicating again that two different response types could be discriminated. The large object most frequently elicited weak turning responses towards the object that partly compensated for the distinct response type with a high probability.

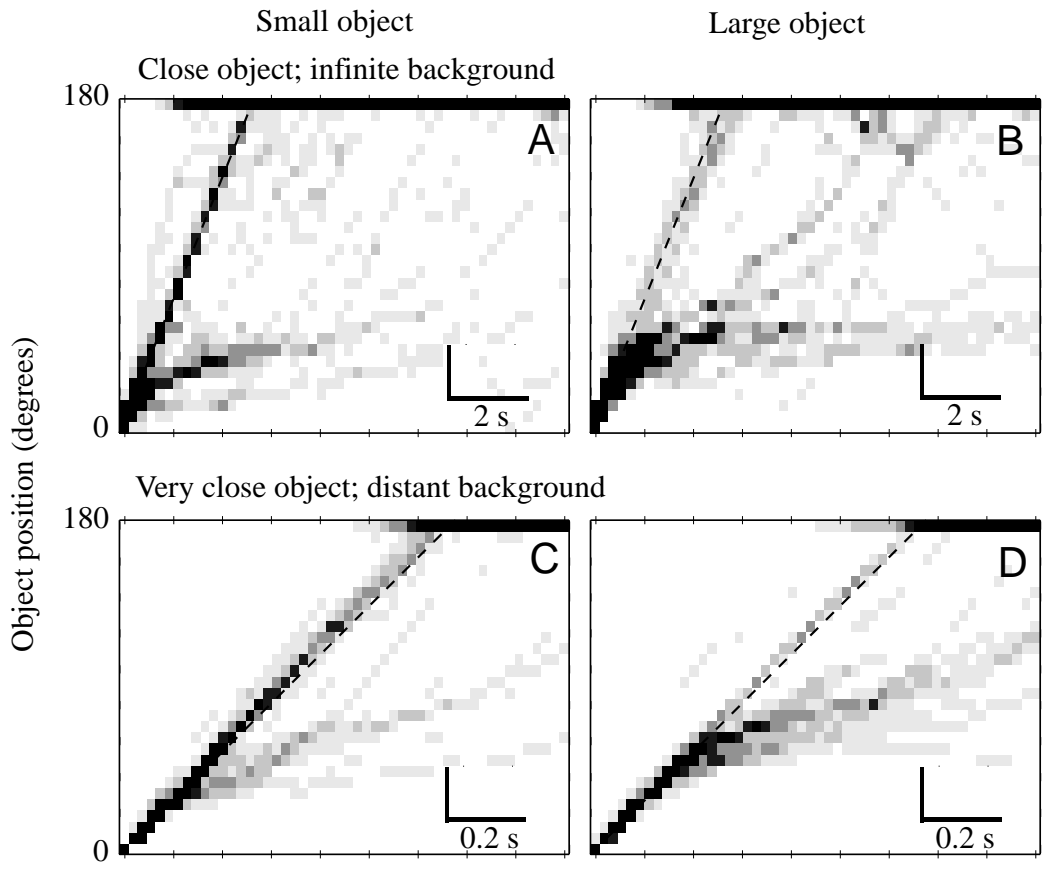

Time (s)

Small object Close object; infinite background
Fig. 6. Spatio-temporal probability distributions of object position for close (A,B) and very close (C,D) objects. The background was simulated to be at infinite distance $(A, B)$ or distant (C,D). Object width was $15^{\circ}$ $(\mathrm{A}, \mathrm{C})$ or $60^{\circ}(\mathrm{B}, \mathrm{D})$. The time resolution was $200 \mathrm{~ms}$ $(A, C)$ or $20 \mathrm{~ms}$ (B,D). For further explanation, see Fig. 5. obvious: either the object moved into the rear part of the arena, starting at a velocity higher than the object's open-loop large object was also fixated frontally over the whole period of $10 \mathrm{~s}$. In contrast to the small object, there was no second 

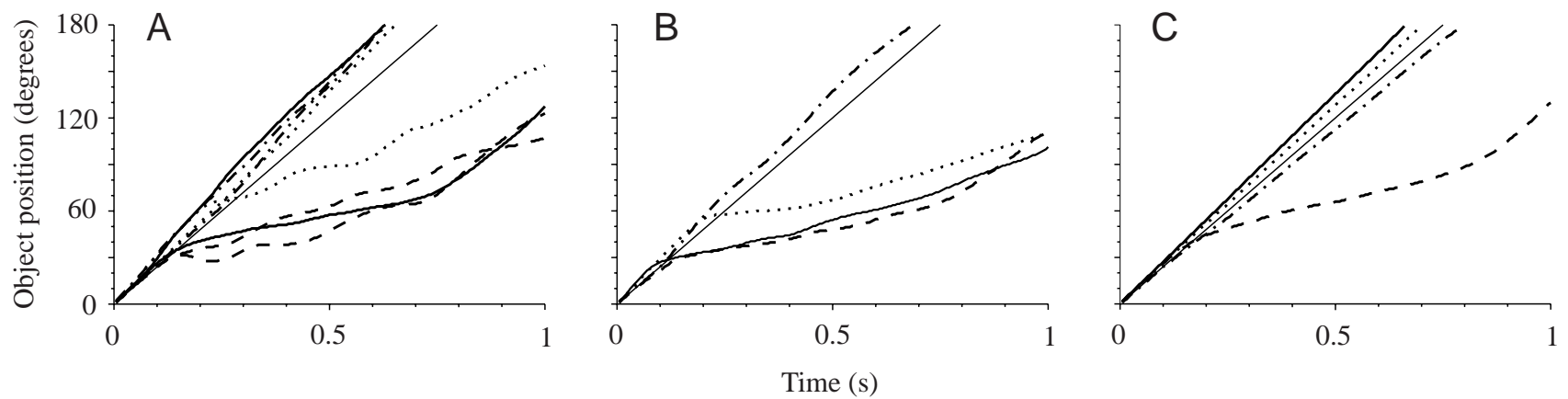

Fig. 7. Time-dependent object fixation by individual flies. Time courses of the position of small objects simulated to be very close. The data were taken from experiments with three different flies (A-C). The time scale starts with object appearance. Background conditions are as follows: stationary (dotted lines), infinitely distant (dot-dash lines), distant (dashed lines) or close (thick solid lines). Thin solid lines show the time course of the position the object would have taken under open-loop conditions. See Materials and methods for definitions of stimulus conditions. In A, each fly performed twice under each of the four stimulus conditions.

object's motion bias but were not sufficient to fixate it at a constant position.

A segregation of the spatio-temporal probability distribution into distinct response types, i.e. fixation and non-fixation, was observed under several stimulus conditions (Fig. 6). When the background was simulated to be at infinite distance and the object was close (Fig. 6A,B), the object could not be fixated in most of the trials and moved into the rear part of the arena, where it remained until the end of the trial. Nonetheless, the flies fixated both the small and the large object in some trials. The large object was fixated more frequently than the small object (see also Fig. 4). When small and large objects were simulated to be very close and the background was distant (Fig. 6C,D), the flies responded in some of the trials with turning reactions towards the object. The responses to the large object started somewhat later and, therefore, at a more lateral position in the visual field than the responses to the small object. Note the different scaling of the time axes in the Fig. 6A,B and Fig. 6C,D.

A distinction into two response types was found not only when analyzing the total of all flies' responses but also within the responses of individual flies. This is shown separately for three flies confronted with a small object simulated to be very close in front of backgrounds at a range of distances (Fig. 7A-C). The object was either fixated or ignored by each of the flies. Thus, by taking into account the time course of object fixation, it was shown that the fly responds to several conditions of relative motion between object and background in either of two ways, object fixation or non-fixation, with intermediate responses being less probable.

\section{Discussion}

Tethered flying flies were exposed to situations simulating translational flights in an environment consisting of an object and its background. The rotational component of the optic flow was determined by the flies' turning reactions, while the translational component was constant and determined by the experimenter. Object and background were simulated to be at different distances from the fly by varying their respective translational velocity. As long as no object was present, the torque fluctuations produced by the fly were smallest when the background was simulated to be distant and much larger when the background was simulated to be very close. Under all conditions apart from those in which the background was very close, the visual feedback of the fly's actions and reactions tended to straighten the flight course. Objects simulated to be close or very close were fixated more readily than distant objects. In most cases, a large object was fixated better than a small one, but at a more lateral position. There was no evidence for object-induced fixation responses when the object was simulated to be more distant than the background, a situation that never occurs under natural conditions. For some of the stimulus conditions, a distinction into either 'no responses' or 'clear fixation responses' was possible. Intermediate responses occurred less often.

\section{How well could natural flight situations be approximated?}

In a flight simulator, it is possible to control the visual stimuli and to assess directly behavioural parameters such as yaw torque. In contrast to free flight, this approach therefore allows the experimenter systematically to analyse stimulus-response relationships. However, in the flight simulator, the stimuli are less complex than those experienced by freely flying animals. In previous studies on object detection and fixation using tethered flying flies, either only rotations of the animal or straight translational flight was simulated, and the behaviour of the fly had little or no influence on the motion stimuli presented (e.g. Virsik and Reichardt, 1976; Reichardt et al., 1983; Egelhaaf, 1985a; Kimmerle et al., 1997). In the present experiments, we therefore attempted to use motion stimuli that shared the following important features with optic flow as experienced during free flight. (i) Turning responses induced counter-rotation of the retinal image (closed-loop situation). (ii) The motion stimuli consisted of a rotational component that was independent of the distance of the object and the background from the fly and of a translational component that depended on distance. Nevertheless, the optic flow to which the flies were subjected in the present 
experiments differed substantially from optic flow as experienced during free flight in the following ways. (i) Pattern motion in the present experiments was always horizontal, whereas during real translation, the optic flow spreads radially from the pole in the heading direction. Moreover, the translational velocity was constant along the azimuth within the right and left visual fields, whereas in a natural situation the translational velocity depends on the visual angle with respect to the heading direction. However, it has been shown that translational motion approximated in a similar way to the present experiments can elicit landing responses (Borst, 1990) and compensatory responses of the thrust force (Götz, 1968). (ii) Object fixation in the flight simulator required sustained turning responses by the fly. Otherwise, the object moved into the rear part of the arena because of its translational motion bias. In contrast, once a fly has turned towards a stationary object and is heading towards it in free flight, no further turning responses are necessary to fixate the object. The interpretations of the turning responses measured in the present study with respect to the simulated distance of the object are therefore, in a strict sense, only valid for the initial fixation period.

\section{Influence of the simulated distance of the object and background from the fly}

It was concluded that objects elicited fixation responses by the fly only if they were simulated to be closer than the background. This is in accordance with a previous study in which relative motion stimuli were presented under open-loop conditions during simulated translational flight (Kimmerle et al., 1997). With respect to visual orientation behaviour in natural environments, this means that flies are only attracted by relative motion cues if they are from objects that are, for instance, elevated above the ground and not from a hole or a dip in the ground.

Objects simulated to be very close were only fixated if the background was non-stationary. It is conceivable from Fig. 4D, that although not statistically significant on the basis of the present data, object fixation may be facilitated when the optic flow contains a translational component. This would corroborate earlier results on object-directed turning behaviour under open-loop conditions (Kimmerle et al., 1997). The deviations from straight flight in the absence of an object were smallest when the background was simulated to be distant. One might therefore speculate that both course control and detection and fixation of close objects are performed most efficiently when the background is at a particular distance from the fly and therefore induces a moderate translational component in the optic flow.

\section{Gating of object fixation}

It was observed under several stimulus conditions that the object was either fixated or that no fixation responses were elicited. Intermediate responses occurred less often. Such a bimodal distribution of responses in the behavioural context of object detection - a full response or no response - suggests a gating mechanism in the neural pathway mediating object fixation. Indications for such a gating mechanism in the objectdetection system of the fly have been reported previously (Zanker et al., 1991). At which stage of the neuronal pathway does gating take place? A central processing stage for optic flow in the visual system of the fly occurs at the large tangential cells in the fly's third visual neuropile, the lobula plate. The tangential cells spatially integrate local motion information over large parts of the visual field and may interact with each other to enhance their selectivity for optic flow (see Hausen and Egelhaaf, 1989). The presumed representatives of the object-detection system at the level of the lobula plate, the figure-detection (FD) cells (Egelhaaf, 1985b; Kimmerle and Egelhaaf, 2000), respond to object motion with some variability. However, the distribution of their response strength is unimodal (B. Kimmerle, unpublished observations). A bimodal response distribution, as found in the motor output, cannot therefore be explained on the basis of the responses of these cells, and the gating mechanism must therefore be assumed to act at a subsequent processing stage. The descending neurons in the lateral protocerebrum form a potential site. Some of these neurons receive, in addition to visual input from the lobula plate, input from the mechanosensory system of the antennae (Gronenberg and Strausfeld, 1990, 1992). Gating of the sensory responses of descending neurons has recently been demonstrated in crickets (Staudacher and Schildberger, 1998). These neurons were shown to be gated by the walking activity of the animal. Gating mechanisms have also been described at the level of motor neurons. Motor neurons supplying the flight-steering muscles in flies have been shown to respond to optomotor stimuli only during flight (Heide, 1983). In the present context, the motor system can be excluded from responsibility for the gating because the flies were flying throughout the experiment.

\section{Object fixation and optomotor course control - processing at the neuronal level}

As mentioned above, optic flow elicited by background and object motion is assumed to be evaluated by the tangential cells in the lobula plate of the fly. The FD cells respond best to small objects and are inhibited during wide-field motion (Egelhaaf, 1985b; Kimmerle and Egelhaaf, 2000); they are therefore believed to play a role in mediating fixation responses. The presumed representatives of the optomotor system for controlling body movements about the vertical axis of the animal are the horizontal system (HS) cells (Hausen, 1982). How specific are the responses of these cell classes to combined object and background motion such as that occurring in a behavioural situation in which the fly has some control over its visual input? In a subsequent study (B. Kimmerle and M. Egelhaaf, in preparation), this question will be investigated by repeating in electrophysiological experiments on both cell types the optic flow generated in the present behavioural experiments during object fixation.

We are grateful to N. Böddeker, R. Kern, H. Krapp, R. Kurtz and A.-K. Warzecha and the anonymous referees for 
critical comments on the manuscript. The help of the departmental electronic and mechanical workshops in designing and building the LED arena is greatfully acknowledged. This study was supported by the Deutsche Forschungsgemeinschaft (DFG).

\section{References}

Borst, A. (1990). How do flies land? From behavior to neuronal circuits. BioScience 40, 292-299.

Egelhaaf, M. (1985a). On the neuronal basis of figure-ground discrimination by relative motion in the visual system of the fly. I. Behavioural constraints imposed on the neuronal network and the role of the optomotor system. Biol. Cybern. 52, 123-140.

Egelhaaf, M. (1985b). On the neuronal basis of figure-ground discrimination by relative motion in the visual system of the fly. II. Figure-detection cells, a new class of visual interneurons. Biol. Cybern. 52, 195-209.

Egelhaaf, M. and Borst, A. (1993). A look into the cockpit of the fly: Visual orientation, algorithms and identified neurons. $J$. Neurosci. 13, 4563-4574.

Egelhaaf, M. and Warzecha, A. (1999). Encoding of motion in real time by the fly visual system. Curr. Opin. Neurobiol. 9, 454-460.

Fermi, G. and Reichardt, W. (1963). Optomotorische Reaktionen der Fliege Musca domestica. Kybernetik 2, 15-28.

Götz, K. G. (1964). Optomotorische Untersuchungen des visuellen Systems einiger Augenmutanten der Fruchtfliege Drosophila. Kybernetik 2, 77-92.

Götz, K. G. (1968). Flight control in Drosophila by visual perception of motion. Kybernetik 4, 199-208.

Götz, K. G. (1975). The optomotor equilibrium of the Drosophila navigation system. J. Comp. Physiol. 99, 187-210.

Gronenberg, W. and Strausfeld, N. J. (1990). Descending neurons supplying the neck and flight motor of Diptera: Physiological and anatomical characteristics. J. Comp. Neurol. 302, 973-991.

Gronenberg, W. and Strausfeld, N. J. (1992). Premotor descending neurons responding selectively to local visual stimuli in flies. $J$. Comp. Neurol. 316, 87-103.

Hausen, K. (1982). Motion sensitive interneurons in the optomotor system of the fly. II. The horizontal cells: Receptive field organization and response characteristics. Biol. Cybern. 46, 67-79.

Hausen, K. and Egelhaaf, M. (1989). Neural mechanisms of visual course control in insects. In Facets of Vision (ed. D. Stavenga and R. Hardie), pp. 391-424. Berlin: Springer Verlag.

Heide, G. (1983). Neural mechanisms of flight control in Diptera. In Biona-Report 2 (ed. W. Nachtigall), pp. 35-52. Stuttgart, New York: Akad. Wiss. Mainz, G. Fischer.
Heisenberg, M. and Wolf, R. (1988). Reafferent control of optomotor yaw torque in Drosophila melanogaster. J. Comp. Physiol. A 163, 373-388.

Kern, R., Egelhaaf, M. and Srinivasan, M. V. (1997). Edge detection by landing honey bees: Behavioural analysis and model simulations of the underlying mechanism. Vison Res. 37, 2103-2117.

Kimmerle, B. and Egelhaaf, M. (2000). Detection of object motion by a fly neuron during simulated flight. J. Comp. Physiol. A $\mathbf{1 8 6}$, 21-31.

Kimmerle, B., Egelhaaf, M. and Srinivasan, M. V. (1996). Object detection by relative motion in freely flying flies. Naturwissenschaften 83, 380-381.

Kimmerle, B., Warzecha, A.-K. and Egelhaaf, M. (1997). Object detection in the fly during simulated translatory flight. J. Comp. Physiol. A 181, 247-255.

Miles, F. A. and Kawano, K. (1987). Visual stabilization of the eyes. Trends Neurosci. 10, 153-158.

Miles, F. A. and Wallman, J. (1993). Visual Motion and its Role in the Stabilization of Gaze. Amsterdam: Elsevier.

Regan, D. and Beverly, K. I. (1984). Figure-ground segregation by motion contrast and by luminance contrast. J. Opt. Soc. Am. A $\mathbf{1}$, 433-442.

Reichardt, W., Poggio, T. and Hausen, K. (1983). Figure-ground discrimination by relative movement in the visual system of the fly. Part II. Towards the neural circuitry. Biol. Cybern. 46 (Suppl.), $1-30$.

Srinivasan, M. V., Lehrer, M. and Horridge, G. A. (1990). Visual figure-ground discrimination in the honeybee: the role of motion parallax at boundaries. Proc. R. Soc. Lond. B 238, 331-350.

Staudacher, E. and Schildberger, K. (1998). Gating of sensory responses of descending brain neurones during walking in crickets. J. Exp. Biol. 201, 559-572.

van Doorn, A. J. and Koenderink, J. J. (1982). Visibility of movement gradients. Biol. Cybern. 44, 167-175.

van Doorn, A. J. and Koenderink, J. J. (1983). Detectability of velocity gradients in moving random-dot patterns. Vision Res. 23, 799-804.

Virsik, R. P. and Reichardt, W. (1976). Detection and tracking of moving objects by the fly Musca domestica. Biol. Cybern. 23, 83-98.

Wolf, R. and Heisenberg, M. (1990). Visual control of straight flight in Drosophila melanogaster. J. Comp. Physiol. A 167, 269-283.

Zanker, J. M., Egelhaaf, M. and Warzecha, A.-K. (1991). On the coordination of motor output during visual flight control of flies. $J$. Comp. Physiol. A 169, 127-134. 